\section{Escape rates for noisy maps with anomalous prefactors}

To cite this article: P. Reimann and E. Lootens 1996 EPL 341

View the article online for updates and enhancements.

\section{Related content}

$$
\begin{aligned}
& \text { - Scaling treatment of the random-field Ising } \\
& \text { model } \\
& \text { - What happens to two-electron resonances } \\
& \frac{\text { when their energy approachesthe break- }}{\text { up threshold? }} \\
& \text { - Strong-pinning phenomena and low- } \\
& \text { temperature heat capacitance anomaly in } \\
& \text { charge and spin-density wave compounds }
\end{aligned}
$$

\section{Recent citations}

- Flux of a Ratchet Model and Applications
$\underline{\text { to Processive Motor Proteins }}$
Li Jing-Hui
- Giant enhancement of activation rate and
$\frac{\text { its corresponding stochastic giant }}{\text { resonance }}$
Jing-hui Li
- Current and efficiency enhancement in
$\frac{\text { Brownian motors driven by non Gaussian }}{\text { noises }}$
S. Bouzat and H. S. Wio

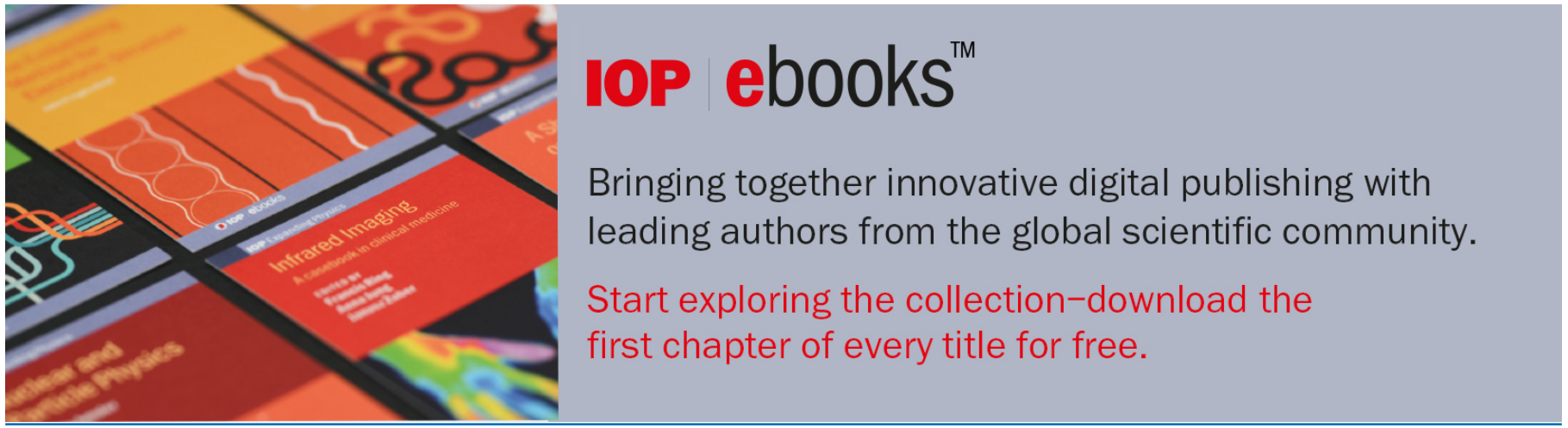

This content was downloaded from IP address 193.190 .2 .250 on 15/09/2020 at 09:18 
Europhys. Lett., 34 (1), pp. 1-6 (1996)

\title{
Escape rates for noisy maps with anomalous prefactors
}

\author{
P. Reimann $(*)$ and E. Lootens \\ Limburgs Universitair Centrum - 3590 Diepenbeek, Belgium
}

(received 28 September 1995; accepted 9 February 1996)

PACS. 05.40+j - Fluctuation phenomena, random processes, and Brownian motion.

PACS. 02.50-r - Probability theory, stochastic processes, and statistics.

\begin{abstract}
The escape rate from a point attractor across an unstable fixed point is studied for a noisy map dynamics in 1 dimension. It is shown that for additive white noise $\xi$ with a distribution proportional to $\exp \left[-|\xi|^{\alpha}\right], \alpha>1$, the escape rate is dominated by an exponentially leading Arrhenius-like factor in the weak-noise limit. However, with the exception of Gaussian noise $(\alpha=2)$, the pre-exponential contribution to the rate still depends more strongly than any power law on the noise strength.
\end{abstract}

Low-dimensional discrete-time models have proven to provide valuable qualitative and even quantitative insight for many systems, though their original setting may be higher dimensional and continuous in time [1]. Specifically, deterministic chaos can be observed already in 1 dimension, offering a relatively easy analytical and numerical investigation of these phenomena. On the other hand, for a satisfactory description of real systems one often has to include also the effect of a small amount of noise. In one-dimensional maps, the prominent role of weak noise near bifurcations, the onset of chaos, crises, or intermittency is well known [1]. From a different point of view, noisy one-dimensional maps are of particular interest as one of the simplest examples that generically violate the so-called condition of detailed balance [2]. Here, we will focus on a further important aspect, namely that weak noise acting on a map with coexisting attractors may render some of them metastable and hence dramatically change the long-time behaviour of trajectories starting inside the corresponding deterministic basins of attraction [3]. We will restrict ourselves to the simplest case of noise-assisted escape from a metastable point attractor across an unstable fixed point, which may be considered as a discrete-time variant of the celebrated Kramers escape problem [4].

We consider the following one-dimensional noisy dynamics of a particle $x$ in discrete time $n$ :

$$
x_{n+1}=f\left(x_{n}\right)+\sigma \xi_{n},
$$

where $f(x)$ is a (sufficiently smooth) map of the real axis with a single stable and unstable fixed point at $x=0$ and $x=1$, respectively. In order to keep things simple only, we further assume that $0<f^{\prime}(x)<1$ for $x \leq 0$ and $f^{\prime}(x)>1$ for $x \geq 1$, implying that, in addition to

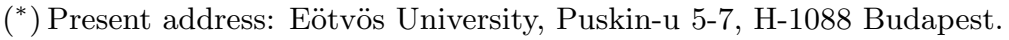

(C) Les Editions de Physique 
the point attractor at $x=0$ with basin $x<1$, there is a second "attractor" at $x=+\infty$ with basin $x>1$. A simple example is the piecewise linear map

$$
f(x)=\max \{s x, 1+u(x-1)\},
$$

where $s$ and $u$ are parameters satisfying $0<s<1$ and $u>1$. The noise $\xi_{n}$ in (1) is given by independent, identically distributed random numbers with probability density

$$
P(\xi)=\frac{\alpha}{2 \Gamma(1 / \alpha)} \exp \left[-|\xi|^{\alpha} \mid\right], \quad \alpha>1,
$$

and the coupling strength $\sigma$ of the noise is assumed to be small. This distribution (3) has become a standard example for studying the effects of white noise with exponentially decaying but not necessarily Gaussian tails on a map dynamics [5].

The point attractor $x=0$ of the deterministic dynamics becomes metastable upon addition of a small amount of noise (1) and the probability distribution of the particles approaches a quasi-invariant density $W(x)$ for large times $n$, satisfying

$$
\int_{-\infty}^{\infty} P(x \mid y) W(y) \mathrm{d} y=(1-k) W(x) .
$$

Here, $k$ is the decay rate of the metastable state $x=0$ and the one-step transition probability $P(x \mid y)$ from $y$ to $x$ is given by $\int_{-\infty}^{\infty} \delta(x-f(y)-\sigma \xi) P(\xi) \mathrm{d} \xi$. Integrating (4), one finally obtains

$$
k=\frac{\int_{-\infty}^{1}\left[W(x)-\int_{-\infty}^{\infty} P(x \mid y) W(y) \mathrm{d} y\right] \mathrm{d} x}{\int_{-\infty}^{1} W(x) \mathrm{d} x}
$$

showing that $k$ can also be interpreted as the escape rate of particles from the basin $x<1$ of the point attractor $x=0$ in the quasi-stationary state.

Next we highlight the calculation of the rate (5) for a particularly simple map $f(x)$, namely the piecewise linear example (2) in the limit $u \rightarrow \infty$. To this end, we first consider the globally linear map $f(x)=s x(x \in \mathbf{R}, 0<s<1)$. In this case, a "true" invariant density $\widetilde{W}(x)$ is reached for large times $n$, satisfying the master equation (4) with $k=0$. It is sufficient to determine a solution $W_{1}(x)$ of this equation (4) for $\sigma=1$ and $x \geq 0$ since obviously $\widetilde{W}(x)=W_{1}(x / \sigma) / \sigma$ and $\widetilde{W}(-x)=\widetilde{W}(x)$. We want to show that for $x \gg 1$ such a solution is given by

$$
W_{1}(x)=Q_{1} x^{\beta \ln x+\gamma_{1}} \exp [-\phi(x)],
$$

where $Q_{1}$ is a normalization constant and

$$
\begin{aligned}
\phi(x) & :=x^{\alpha}\left[1-s^{\frac{\alpha}{\alpha-1}}\right]^{\alpha-1}, \\
\beta & :=\frac{(2-\alpha)(\alpha-1)}{4 \ln s^{-1}}, \\
\gamma_{1} & :=\frac{\alpha-2}{4}+\frac{\alpha-1}{2 \ln s^{-1}} \ln \left(\frac{\alpha \pi \phi(1)^{\frac{2-\alpha}{\alpha-1}}}{2(\alpha-1) \Gamma(1 / \alpha)^{2}}\right),
\end{aligned}
$$

see also fig. 1. To demonstrate the validity of this approximation, we note that for large $x$ the integral in (4) is dominated by the exponentially leading part $\exp [-\psi(x, y)]$ of $P(x \mid y) W_{1}(y)$, where $\psi(x, y):=|x-s y|^{\alpha}+\phi(y)$. This exponent $\psi(x, y)$, considered as a function of $y$, takes 


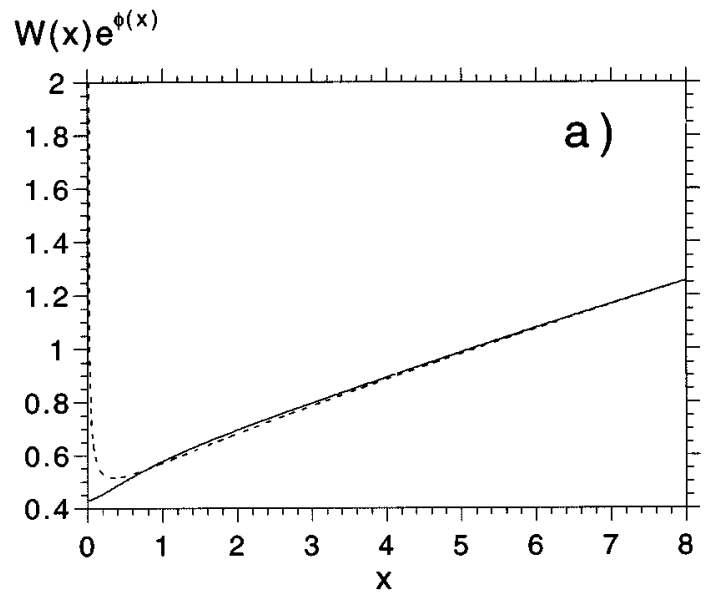

$$
W(x) e^{\phi(x)}
$$

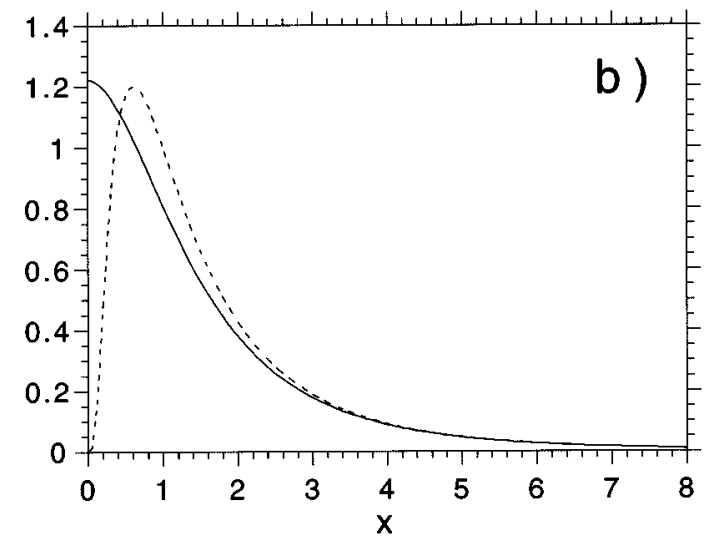

Fig. 1. - Numerical solution $W_{1}(x)$ of the master equation (4) with $f(x)=s x, k=0$ and $\sigma=1$ at the parameter values $s=1.5, \alpha=1.5(a))$ and $s=0.5, \alpha=3(b))$. The solid lines show these numerical solutions $W_{1}(x)$ multiplied by $\exp [\phi(x)]$ according to $(8)$. The dashed lines are the corresponding asymptotic approximations (6) with $Q_{1}=0.57$ in $a$ ) and $Q_{1}=0.43$ in $b$ ).

its absolute minimal value $\phi(x)$ at $y=g(x):=x s^{1 /(\alpha-1)}$ and can be expanded about this minimum as

$$
\phi(x)+\Delta y^{2} \frac{\alpha(\alpha-1) x^{\alpha-2}}{2(\phi(1) s)^{\frac{2-\alpha}{\alpha-1}}}+O\left(\Delta y^{3} x^{\alpha-3}\right),
$$

where $\Delta y:=y-g(x)$. Thus, for large $x$ the main contributions to the integral (4) stem from a region about $y=g(x)$ with the extension of a few $x^{1-\alpha / 2}$. In this region the term $O\left(\Delta y^{3} x^{\alpha-3}\right)$ in (10) and the variation of the pre-exponential factor $Q_{1} y^{\beta \ln y+\gamma_{1}}$ of $W_{1}(y)$ (see (6)) are negligible. A straightforward calculation then shows that (6) is indeed a consistent solution of the master equation (4) (with $k=0, \sigma=1$ ) for $x \gg 1$. Note that the normalization factor $Q_{1}$ in (6) depends on $\alpha$ and $s$, but obviously not on $\sigma$. However, its explicit value cannot be determined analytically except for Gaussian noise $(\alpha=2)$, see also ref. [6] and fig. 1 .

We now return to the piecewise linear map (2) in the limit $u \rightarrow \infty$. It is not difficult to verify [3] that the numerator of the escape rate (5) can be rewritten as a flux of particles $\int_{1}^{\infty} \mathrm{d} x \int_{-\infty}^{1} \mathrm{~d} y P(x \mid y) W(y)$ from $[-\infty, 1]$ into $[1, \infty]$ minus a back-flow $\int_{-\infty}^{1} \mathrm{~d} x \int_{1}^{\infty} \mathrm{d} y$. $P(x \mid y) W(y)$ from $[1, \infty]$ into $[-\infty, 1]$. The latter becomes negligible for $u \rightarrow \infty$, implying that

$$
k=\frac{\int_{1}^{\infty} \mathrm{d} x \int_{-\infty}^{1} \mathrm{~d} y P(x \mid y) W(y)}{\int_{-\infty}^{1} \mathrm{~d} x W(x)} .
$$

Further, one can show by a similar line of reasoning as in the preceding paragraph that in the domain $-\infty<x<1$, the quasi-invariant density $W(x)$ can be approximated by the "true" invariant density $\widetilde{W}(x)$ associated to the globally linear map. Hence, the denominator in (11) asymptotically approaches 1 for small $\sigma$ and in the numerator one can substitute $W(x)$ by $W_{1}(x / \sigma) / \sigma$. Using (6), a straightforward calculation then yields

$$
k=Q \sigma^{\beta \ln \sigma+\gamma} \exp \left[-\phi(1) / \sigma^{\alpha}\right],
$$

where $\gamma:=\alpha-1-\gamma_{1}$ and $Q$ is asymptotically $\sigma$-independent but explicitly known only for Gaussian noise [6]: $Q=\left[4 \pi\left(1-s^{2}\right)\right]^{-1 / 2}$ for $\alpha=2$. 

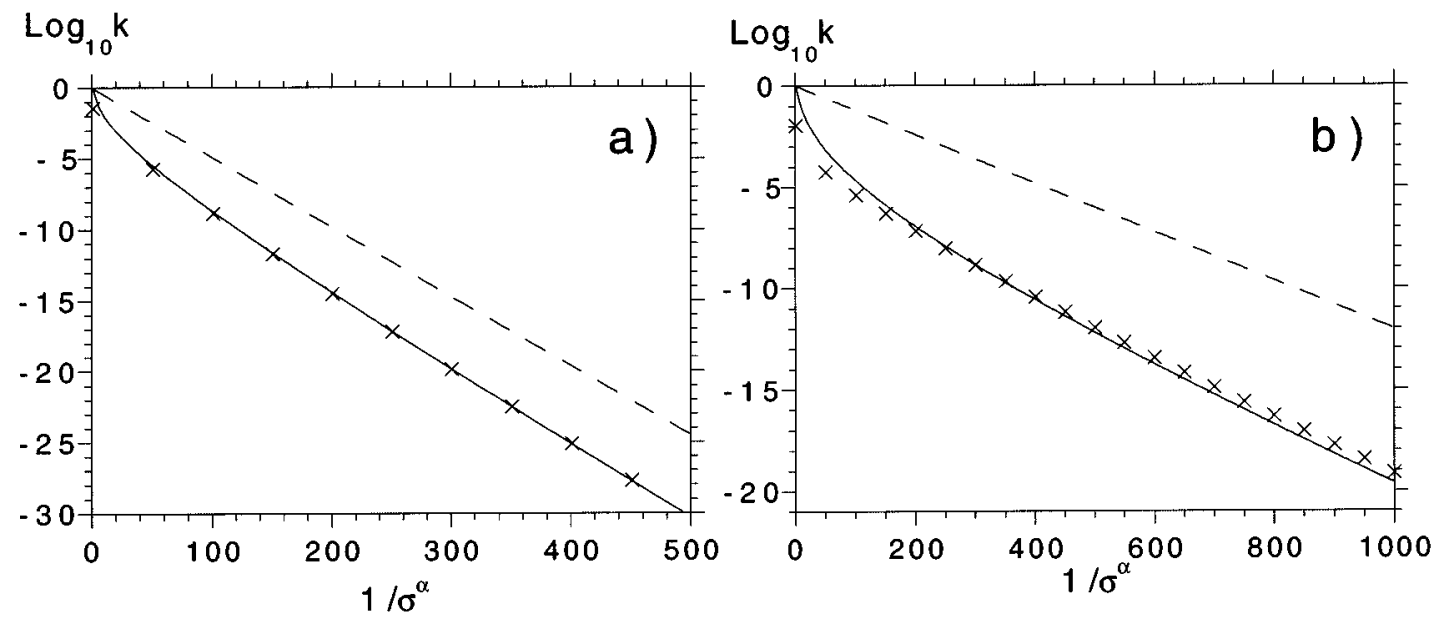

Fig. 2. - Arrhenius plot of the numerical rate (crosses) determined according to (4), (11) for the piecewise linear map (2) with $s=0.5, u \rightarrow \infty(a))$ and $s=0.5, u=2(b))$. The noise distribution is given by (3) with $\alpha=5$. The numerical uncertainty is a few percent. The solid and dashed lines represent the theoretical estimates $\sigma^{\beta \ln \sigma+\gamma} \exp \left[-\phi(1) / \sigma^{\alpha}\right]$ and $\exp \left[-\phi(1) / \sigma^{\alpha}\right]$, respectively, see (12).

Thus, the escape rate (12) is exponentially dominated by the Arrhenius-like factor $e^{-\phi(1) / \sigma^{\alpha}}$. However, for non-Gaussian noise $(\alpha \neq 2)$ the pre-exponential "correction" $Q \sigma^{\beta \ln \sigma+\gamma}$ still depends more strongly than any power law on the noise strength $\sigma$. To the best of our knowledge, such an extreme behaviour of the prefactor in a Kramers-like escape problem [4] is observed here for the first time, see fig. $2 a$ ). Note that the limit $s \rightarrow 0$ is not admitted in (12) unless $\alpha=2$ since it does not commute with the limit $x \rightarrow 0$, in which the approximation (6) becomes exact. Similarly, one can see that the limit $\alpha \rightarrow 1$ is not admitted.

We finally sketch the extension of our approach for a general noisy map (1). Close to the stable fixed point $x=0$, the quasi-invariant density $W(x)$ can be approximated by the solution $\widetilde{W}(x)$ for the corresponding globally linear map. In particular, $W(x)=W_{1}(x / \sigma) / \sigma$ follows from (6) for $\sigma \ll x \ll 1$ and symmetrically for negative $x$. For more general $x$, we adopt a WKB-ansatz $W(x)=Z(x) \exp \left[-\phi(x) / \sigma^{\alpha}\right]$, where the so-called generalized potential $\phi(x)$ is required to satisfy the functional equation $[2]$

$$
\phi(x)=\min _{y}\left\{\phi(y)+|x-f(y)|^{\alpha}\right\} .
$$

To make $\phi(x)$ unique, we further require that $\phi(0)=0$. It turns out [2], [6] that there still remain infinitely many different solutions of (13), each being uniquely characterized by its value at the unstable fixed point $x=1$. Within our WKB-ansatz the appropriate choice is to select the one with the largest value of $\phi(1)$. For piecewise linear maps, as for instance (2), this solution of (13) can be obtained analytically, while for more general $f(x)$ approximation schemes and powerful numerical methods are available [2], [3], [6]. Once the generalized potential $\phi(x)$ is known, the prefactor $Z(x)$ can be determined recursively by means of a saddle-point approximation in the master equation (4), provided the noise strength $\sigma$ is sufficiently small. In this recursion relation, the above-mentioned approximation for $W(x)$ in the domain $\sigma \ll x \ll 1$ is needed as initial condition. However, close to the unstable fixed point $x=1$ the saddle-point approximation and hence the recursion relation are no longer valid. In this region, one has to determine a proper local solution of the full master equation (4) which correctly matches the already known solution $W(x)$ outside this region. For Gaussian noise 
$(\alpha=2)$, these concepts have been elaborated in [6]. For $\alpha \neq 2$, the calculations become more involved. The main steps follow by combining the line of reasoning from [6] and [7].

Given the quasi-invariant density $W(x)$, the evaluation of the escape rate (5) is straightforward. One recovers exactly the same result as in (12), except that now $\beta$ is of the form

$$
\beta=\frac{(2-\alpha)(\alpha-1)}{4 \ln f^{\prime}(0)^{-1}}+\frac{(2-\alpha)(\alpha-1)}{4 \ln f^{\prime}(1)},
$$

whereas $\gamma$ now depends on $\alpha$ and on global properties of the map $f(x)$ but not on $\sigma$. Similarly to $\phi(1)$, the explicit value of $\gamma$ can be determined analytically only in special cases. An example is the piecewise linear map (2) for which one finds after a rather lengthy calculation that

$$
\begin{aligned}
\phi(1) & =\left(\frac{\left(1-s^{\frac{\alpha}{\alpha-1}}\right)\left(u^{\frac{\alpha}{\alpha-1}}-1\right)}{u^{\frac{\alpha}{\alpha-1}}-s^{\frac{\alpha}{\alpha-1}}}\right)^{\alpha-1}, \\
\gamma & =\frac{\alpha}{2}-\left(\frac{\alpha-1}{2 \ln s^{-1}}+\frac{\alpha-1}{2 \ln u}\right) \ln \left(\frac{\alpha \pi \phi(1)^{\frac{2-\alpha}{\alpha-1}}}{2(\alpha-1) \Gamma(1 / \alpha)^{2}}\right),
\end{aligned}
$$

see fig. $2 b$ ). In addition to the limits $s \rightarrow 0$ and $\alpha \rightarrow 1$ (cf. the discussion after eq. (12)) also $u \rightarrow \infty$ is not admitted here unless $\alpha=2$. Finally, for Gaussian noise $(\alpha=2)$ one can show [6] that $\gamma=1$ for arbitrary $f(x)$ and that $Q=[4 \pi \phi(1)]^{-1 / 2}$ for the piecewise linear map (2).

In summary, we have demonstrated that for a large class of very simple noisy maps (1), (3) the escape rate (12) is dominated for small $\sigma$ by an exponentially leading Arrhenius factor. However, the pre-exponential contributions to the rate still depend more strongly than any power law on $\sigma$ whenever $\alpha \neq 2$, see eq. (14). Therefore, this prefactor is not at all a negligible "correction" in many cases: For instance, when the true escape rate is about $10^{-8}$, the leading-order estimate $\exp \left[-\phi(1) / \sigma^{\alpha}\right]$ may still differ by more than a factor 1000 , see fig. 2 .

One can show [2] that the stochastic process (1), (3) with Gaussian noise $\alpha=2$ fulfils the condition of detailed balance if and only if $f(x)$ is a globally linear map, $f(x)=s x$, satisfying $-1<s<1$. Similarly, for non-Gaussian noise $\alpha \neq 2$, detailed balance is found to hold only for the identically vanishing map $f(x) \equiv 0$. This explains why even the globally linear map $f(x)=s x$ with $s>0$ turned out to be a non-trivial problem for non-Gaussian noise.

It is possible to extend our approach to much more general maps $f(x)$ than those specified below eq. (1). Not only one can of course relax the conditions on $f^{\prime}(x)$, but also maps with periodic or chaotic attractors and repellers may be included [7]. Further, a large class of multiplicative couplings of the noise in (1) is also admissible. While the technical difficulties considerably increase, the general form (12) of the escape rate remains unchanged in all these cases.

$$
* * *
$$

We tank A. Hamm for helpful discussions. Financial support by the Program on InterUniversity Attraction Poles of the Belgian Government, the IIKW Belgium, and the Holderbank foundation (Switzerland) is gratefully acknowledged.

\section{REFERENCES}

[1] For a review see Schuster H. G., Deterministic Chaos (VHC Verlagsgesellschaft, Weinheim) (1988).

[2] Reimann P. and Talkner P., Phys. Rev. A, 44 (1991) 6348. 
[3] Talkner P., Hänggi P., Freidkin E. and Trautmann D., J. Stat. Phys., 48 (1987) 231; TAlkner P. and HängGi P., in Noise in Nonlinear Dynamical Systems, edited by F. Moss and P. V. E. McClintock, Vol. 2 (Cambridge University Press, Cambridge) 1989.

[4] For reviews see: Hänggi P., Talkner P. and Borkovec M., Rev. Mod. Phys., 62 (1990) 251 and New Trends in Kramers' Reaction Rate Theory, edited by P. TALKNER and P. HÄNGGI (Kluwer Academic Publishers, Dordrecht) 1995.

[5] Hamm A. and Graham R., J. Stat. Phys., 66 (1992) 689; Phys. Rev. A, 46 (1992) 6323; Reimann P. and Talkner P., Helv. Phys. Acta, 66 (1993) 93; Hamm A., TÉl T. and Graham R., Phys. Lett. A, 185 (1994) 313; Reimann P., Z. Naturforsch. A, 49 (1994) 1248.

[6] Reimann P. and Talkner P., Phys. Rev. E, 51 (1995) 4105 and contribution in New Trends in Kramers' Reaction Rate Theory, edited by P. TALKNER and P. HäNGGI (Kluwer Academic Publishers, Dordrecht) 1995.

[7] Reimann P., Noisy One-dimensional Maps Near a Crisis, part II, submitted to J. Stat. Phys. 